seconds, including 6 with averages shorter than 10 seconds. The efficacy of hand washing is based on 30- to 60 second protocols, and there is no evidence evaluating the effectiveness of hand washes that last 7 to 10 seconds. The time demand and inconvenience of repeated hand washing; poor access to handwashing facilities, such as a lack of sinks or sinks that are physically blocked by equipment in patient rooms; and the desire to prevent dermatitis, which can develop after frequent hand washing, contribute to the low compliance with handwashing protocols observed in most healthcare facilities.

Alcohol rubs take less time than washing and effectively reduce microbial loads (although washing is necessary to remove visible soil). Alcohol rubs also provide improved access, as there is no dependence on sinks and plumbing, and improved tolerance, as they can be less irritating to hands than soap and water.

The OSHA Bloodborne Pathogens Standard already requires that $\mathrm{HCWs}$ wash hands after removal of gloves and after any potential contact with blood or body fluids, requirements the $\mathrm{HICPAC}$ guideline need not reiterate, committee members said.

In related news, HICPAC's 2001 Guideline for Environmental Controls in Healthcare Facilities is slated for submission to the Federal Register by the end of November.

From: Jacobson A. icanNEWS. November 7, 2000.

\section{VRE in Children With Bone-Marrow Transplants}

The emergence of vancomycin-resistant enterococci (VRE) as nosocomial pathogens is a major problem in the United States; in Europe, VRE nosocomial infections are uncommon and only rarely have been reported in pediatric or neonatal units. Carretto and colleagues from Pavia, Italy, conducted a study to report on the clinical and microbiological features of VRE infections in three children given hematopoietic stem cell transplantation (HSCT). Five episodes of vancomycin-resistant Enterococcus faecium (VREF) infection were diagnosed in three children given an allogeneic HSCT. Molecular methods, such as randomly amplified polymorphic DNA (RAPD) fingerprinting and automated ribotyping, were used to define the circulation of strains.

All of the isolates were resistant to all commercially available agents and showed the vanA genotypic profile. All children were treated successfully with the combination of quinupristin-dalfopristin (QD) plus teicoplanin (TEC), although treatment was not sufficient to eradicate the microorganism promptly from the gastrointestinal tract. All children remain alive. After the first isolation of VRE, a surveillance protocol was started, and they documented that the rate of colonization in children and their mothers was less than $1.5 \%$. The RAPD method demonstrated the possible nosocomial transmission of one strain.
The authors' experience demonstrates that VRE infection is a life-threatening complication in children given HSCT. Prompt diagnosis of this infection and its treatment with the combination of QD and TEC can successfully manage this severe infection in profoundly immunocompromised patients.

FROM: Carretto E, Barbarini D, Locatelli F, Giraldi E, Pellegrini N, Perversi L, et al. Vancomycin-resistant Enterococcus faecium infection in three children given allogeneic hematopoietic stem cell transplantation: clinical and microbiologic features. Haematologica 2000;85:1158-1164.

\section{Outbreak of $B$ cereus Infections in an NICU}

In 1998, an outbreak of systemic infections caused by Bacillus cereus occurred in the neonatal ICU of the University Hospital Vrije Universiteit, Amsterdam, The Netherlands. Three neonates developed sepsis with positive blood cultures. One neonate died, and the other two neonates recovered. Van Der Zwet and colleagues performed an environmental survey, a prospective surveillance study of neonates, and a case-control study in combination with molecular typing to identify potential sources and transmission routes of infection.

Genotypic fingerprinting by amplified-fragment length polymorphism (AFLP) showed that the three infections were caused by a single clonal type of $B$ cereus. The same strain was found in trachea aspirate specimens of 35 other neonates. The case-control study showed mechanical ventilation with a ventilation machine to be a risk factor for colonization or infection (odds ratio, 9.8; 95\% confidence interval, 1.1-88.2). Prospective surveillance showed that colonization with $B$ cereus occurred exclusively in the respiratory tract of mechanically ventilated neonates. The epidemic strain of $B$ cereus was found on the hands of nursing staff and in balloons used for manual ventilation. Sterilization of these balloons ended the outbreak.

The authors concluded that $B$ cereus can cause outbreaks of severe opportunistic infection in neonates. Typing by AFLP proved very useful in the identification of the outbreak and in the analysis of strains recovered from the environment to trace the cause of the epidemic.

FROM: Van Der Zwet WC, Parlevliet GA, Savelkoul PH, Stoof J, Kaiser AM, Van Furth AM, et al. Outbreak of Bacillus cereus infections in a neonatal intensive care unit traced to balloons used in manual ventilation. $J$ Clin Microbiol 2000;38:4131-4136.

\section{Coccidioidomycosis Outbreak: CDC Advisory}

A recent outbreak of coccidioidomycosis (CM) among a Pennsylvania church group who did construction work in Mexico indicates a need for healthcare providers to be alert for this disease in returning travelers who have 
influenza-like symptoms, said the CDC in the November 10, 2000, issue of the Morbidity and Mortality Weekly Report. CM is a fungal disease endemic in the southwestern United States and parts of Central and South America. The disease is acquired by inhaling the spores of Coccidioides immitis, present in soil. Symptoms are usually mild and influenza-like.

Twenty-seven of 35 church members became ill last winter within 2 weeks after returning from Hermosillo, Mexico, where they had spent a week building a church. Initial testing of acute serum specimens by the CDC showed that 1 member had antibodies to $C$ immitis. Subsequently, serum samples and questionnaires were obtained for 30 of the 35 travelers. Of these, 23 reported they had gotten sick in Mexico or after returning home. Serological testing indicated that 8 people, 7 of whom were symptomatic, met the case definition for $\mathrm{CM}$. The most common symptoms were fatigue, fever, and joint and muscle pain; 3 people had a rash, and 4 had a cough. One traveler was hospitalized for a day, and 11 missed work or school (an average of 5.5 days). Eighteen people sought treatment for the illness, and 11 received medications, such as fluconazole or itraconazole.

No activities other than the travel and construction work were associated with the illness. Twenty-two of the church members reported working in very dusty conditions. Approximately $40 \%$ of persons infected with $C$ immi$t i s$ experience symptoms, usually mild and influenza-like; however, $8 \%$ of patients may develop severe pulmonary disease requiring hospitalization, and $7 \%$ develop disseminated extrapulmonary disease. Risk factors for severe pulmonary disease include diabetes, smoking, and older age, whereas risk factors for disseminated disease include black or Asian race, pregnancy, and immunocompromising conditions.

Although avoiding activities that generate dust or using a mask during these activities is advisable, these measures do not provide complete protection from CM. A potential strategy for adequate prevention is vaccine development, because natural infection with $C$ immitis provides lifelong immunity. However, until a vaccine becomes available, organizations that conduct trips to areas where $\mathrm{CM}$ is endemic should inform their travelers about the risks for $\mathrm{CM}$. Healthcare providers should consider CM in travelers returning from areas where the disease is endemic and who present with an influenza-like illness.

FROM: icanNEWS. November 10, 2000.

\section{Community-Acquired MRSA in Queensland, Australia}

Community-acquired methicillin-resistant Staphylococcus aureus (MRSA) susceptible to gentamicin has been reported in a number of countries in the 1990s. To study the acquisition of gentamicin-sensitive (GS) MRSA in southeast Queensland and the relatedness of GS MRSA to other strains of MRSA, 35 cases of infection due to GS MRSA from October 1997 through September 1998 were examined retrospectively to determine the mode of acquisition and risk factors for MRSA acquisition.

Thirty-one isolates from the cases were examined using a variety of methods (antibiotyping, phage typing, pulsed-field gel electrophoresis [PFGE] fingerprinting, and coagulase typing by restriction analysis of polymerase chain reaction products) and were compared with strains of local hospital-acquired GS MRSA and of Western Australian (WA) MRSA. Only 6 of 23 cases of communityacquired GS MRSA had risk factors for MRSA acquisition. Twenty of 21 isolates from cases of community-acquired infection were found to be related by PFGE and coagulase typing and had similar phage-typing patterns. Hospital- and nursing home-acquired GS MRSA strains were genetically and phenotypically diverse. Community-acquired GS MRSA strains were not related to nosocomial GR MRSA or WA MRSA, but phage-typing results suggest they are related to GS MRSA previously reported in New Zealand.

FROM: Nimmo GR, Schooneveldt J, O'Kane G, McCall B, Vickery A. Community acquisition of gentamicinsensitive methicillin-resistant Staphylococcus aureus in southeast Queensland, Australia. J Clin Microbiol 2000;38: 3926-3931.

\section{On-Line Lectures With International Focus}

\section{Judene Bartley}

The Supercourse: Epidemiology, the Internet, and Global Health web site offers a series of lectures on epidemiology and the Internet in English, Spanish, and Japanese for students of medicine and health. The British Medical Journal describes the course as the Global Health Network University. The Supercourse has 1,700 faculty from 110 countries who created a Library of Lectures, with 214 lectures currently available.

The lectures are grouped under the headings New, Revised; Epidemiology and Biostatistics; and Internet and Global Health. Examples of topics include "A Brief Introduction to Epidemiology," "Antibiotics Use and Medical Drug Policy," "Epidemiology of Disasters," "Introduction to Program Evaluation," "Environmental Epidemiology," and "Evidence-Based Medicine: Can We Practice Effectively Without it?" Lectures on specific diseases include, but are not limited to, malaria, cancer, diabetes, and hepatitis. After linking to the index, viewers may simply click on topics and move through the slides and text at their own pace. Hyperlinks within slides permit instant access to sites with related, in-depth information. Interested parties are welcomed to join and either share or review lectures on preferred topics. Go to http:// www.pitt.edu/ super1/. 